mit starkem Zuzug; es wird zwischen drei bis acht Betten auf iooo Einwohner schwanken.

Die Baukosten einer modernen Krankenanstalt werden pro Bett auf 68000 Mark berechnet. Dazu kommen die hohen Betriebskosten, zumal die Gemeindekrankenhuser durchweg Zuschuanstalten sind, bei denen der Zuschu z. B. in Berlin im Jahre 1911 ca. 70\% der Ausgaben betrug. Die vielerorts durch den Zuwachs der Bevlkerung und die erhhte Inanspruchnahme der Krankenanstalten notwendige Vermehrung oder Erweiterung der Krankenhuser ist dann billiger zu gestalten, wenn sog.

Leichtbauten errichtet werden; in diesen werden diejenigen Kranken verpflegt, die nicht oder nicht mehr alle Einrichtungen des modernen Krankenhauses bentigen. Auch werden durch eine Verkrzung der Krankenhausund berfhrung in ambulante Behandlung Betten gespart werden knnen. In Universittsinstituten wird diese Behandlung in Polikliniken ausgefhrt. Die Krankenhuser gehren meistens den Gemeindeverbnden oder der Kirche und religisen Genossenschaften, whrend der Staat nur Trger der Universittskliniken ist, der Ausbildungssttte fr die rzte. Daneben bestehen auch eine Anzahl privater Krankenhuser. ber die gesetzlichen Bestimmungen hinsichtlich des Baues, der Einrichtung, Leitung und Beaufsichtigung wird auf die frhere Besprechung (S. $103 \mathrm{ff}$.) verwiesen.

\title{
2. Sieche.
}

Die Siechen werden in Abteilungen der allgemeinen Krankenhuser oder in besonderen Siechenhusern untergebracht, frher auch als Stift bezeichnet. Sie sind meistens Stiftungen, in die sich alleinstehende Leute fr das Alter einkaufen. Sie stehen jetzt grtenteils in der Verwaltung der Gemeinden oder von Ordensgemeinschaften und haben einen Arzt und Pflegepersonal zur Verfgung. Die modernen Siechenhuser sind krankenhaushnlich eingerichtet und haben besondere Abteilungen $\mathrm{fr}$ Schwerkranke, auch Operationsgelegenheit. Die vorher erwhnte berlastung der allgemeinen Krankenhuser hat auch dazu gefhrt, chronisch Kranke den Siechenhusern zu berweisen, in denen fr eine ausreichende rztliche Behandlung gesorgt ist.

IV. Krankenbefrderungs* und Rettungswesen.

Auf Veranlassung des Zentralkomitees fr das Rettungswesen in Preuen wurden im Jahre 1912 von dem Reichskanzler den Bundesregierungen Grundstze fr die Ordnung des Rettungs- und Krankentransportwesens 
bermittelt, die von den Regierungen den beteiligten Kreisen

weitergegeben wurden1).

1) Preu. Erl. d. Min. d. Inn. vom io. 12. 1912.

177

1. Die Krankenbefrderung haben die Gemeinden entweder der

Feuerwehr, dem Fuhrpark oder der Polizei angegliedert oder einem zuverlssigen

Unternehmer bertragen. In kleineren Stdten und auf dem Lande

ist die Krankenbefrderung zweckmigerweise mit den Krankenhusern

in Verbindung gebracht. Auf dem Lande werden Tragbahren, die meist

fahrbar sind, benutzt ; schon in kleineren Orten sind besondere Wagen mit

Tragbahren bereitgestellt; in den Stdten werden fast ausnahmslos jetzt

Automobile benutzt, die dank ihrer einfachen Innenausstattung leicht zu

desinfizieren sind. Fr den Vollzug der Krankenbefrderung kommen

Krankenpflegepersonen, Mitglieder des Roten Kreuzes oder von Samaritervereinen oder andere, von rzten dazu ausgebildete Personen in Betracht.

Bei Verdacht von bertragbaren Krankheiten ist sowohl fr das Begleitpersonal

als auch fr andere spter zu befrdernde Kranke der ntige

Schutz durch geeignete Desinfektionsmanahmen zu schaffen.

2. Das ettungswesen bezweckt die erste Hilfe bei Unfllen und

pltzlichen Erkrankungen. Neben der Mitarbeit der rzteschaft werden

rztlich berwachte Nothelfer die erste Hilfe leisten. Wo ein Bedrfnis

vorliegt, ist ein stndiger rztlicher Wachdienst eingerichtet; fr jede

Rettungswache mu mindestens ein berufsmiger Nothelfer zur Verfgung

stehen. Von besonderer Bedeutung ist ein gut funktionierendes

Meldewesen, das den rtlichen Verhltnissen angepat sein und jeden vermeidbaren

Zeitverlust ausschlieen mu; es wird sich hauptschlich des

Telefons und des Feuermelders bedienen. Aber auch bei Theater- oder

anderen Vorstellungen oder groen Versammlungen, bei denen mit Unfllen

zu rechnen ist, sollen ausgebildete Samariter mit Verbandsksten und

Arzneimitteln zur Stelle sein. Alle Einrichtungsgegenstnde der Rettungswache

sind stets in Ordnung zu halten und auf ihre Brauchbarkeit zu prfen.

Da die Arzneimittel von Zeit zu Zeit zu erneuern sind, braucht wohl

nicht besonders betont zu werden.

Grere Betriebe und vor allem Bergwerke haben ihre eigene Rettungswache.

Der staatliche Medizinalbeamte bt unter Beteiligung des Kommunalarztes

die Aufsicht ber das gesamte Rettungswesen aus und hat darauf

zu achten, da die ntigen Krankentransportmittel fr die Bevlkerung

.zur Verfgung stehen. 
V. Frsorge fr Blinde, Taubstumme

und Schwerhrige.

In Deutschland gibt es, auf je iooooo Einwohner berechnet, 61 Blinde und 173 Taubstumme. Voraussetzung fr die Frsorgemanahmen ist die statistische Erfassung dieser Kranken, die durch regelmige Erhebungen bei den Blinden und Taubstummen gewhrleistet wird. Beamtete rzte haben die Untersuchung der Kinder vorzunehmen und Formulare nach dem vor geschriebenen Muster auszufllen1).

1) Pr.Ges. vom 7. 8. 11 nebst Ausf.Bestim. vom 21. 12. 1911.

Xustig, Der Arzt als Gesundheitsbeamter. 12 